\section{Slipping therapeutics to the mitochondria}

\section{By Tracey Baas, Senior Editor}

Researchers from The University of Georgia have developed a polymeric nanoparticle technology that can selectively deliver small molecules to the mitochondria of cultured cells. ${ }^{1}$ The researchers now are evaluating the nanoparticles in animal models of Alzheimer's disease, cancer and metabolic disorders.

Mitochondrial dysfunction plays a role in multiple diseases, including cancer, neurodegenerative and neuromuscular diseases, obesity and diabetes. However, targeting mitochondria has been difficult because there are no platforms to deliver drugs directly into the organelles, and nonspecific delivery can lead to toxicity in other parts of the cell.

Thus, two University of Georgia researchers, graduate student Sean Marrache and Assistant Professor of Chemistry Shanta Dhar, reasoned that mitochondria-targeting nanoparticles could be a way to selectively "The two advantages of these
nanoparticles over others are
the fact they are composed of
clinically validated materials,
PLGA and PEG, and are
produced using a single-step
processing method."
-Omid Farokhzad,
Harvard Medical School

Collectively, the data suggested the nanoparticles specifically targeted the mitochondria and that trafficking could be optimized by varying diameter and surface charge.

The scientists also tested the nanoparticles' ability to deliver payloads in cell culture and to alter disease phenotypes.

In human neuroblastoma cells treated with $\beta$-amyloid $(A \beta)$ to model $\mathrm{AD}$, curcumin-loaded, targeting nanoparticles increased cell viability compared with empty targeting nanoparticles or curcumin alone. Curcumin inhibits $A \beta$ and the associated mitochondrial oxidative stress.

In human cervical cancer cells, lonidamine-loaded, targeting nanoparticles increased cell death compared with loaded, nontargeted nanoparticles or free compound, showing a fivefold lower $\mathrm{IC}_{50}$ value than the nontargeting nanoparticles. Lonidamine inhibits mitochondrial glycolysis.

Finally, in preadipocytes cultured under differentiation conditions to model metabolic disease, 2,4-dinitrophenol (2,4-DNP)-loaded, targeting nanoparticles suppressed preadipocyte differentiation and decreased lipid accumulation compared with the loaded, nontargeted nanoparticles or free compound, and did not affect cell viability.

The mitochondrial decoupler 2,4-DNP underwent clinical testing by Stanford University researchers as a weight loss agent in the 1930s but proved toxic at high doses.

Results were reported in the Proceedings of the National Academy of Sciences.

"The two advantages of these nanoparticles over others are the fact they are composed of clinically validated materials, PLGA and PEG, deliver therapies.

The duo conjugated a poly(lactic-co-glycolic acid) (PLGA) and polyethylene glycol (PEG) copolymer to a triphenylphosphonium (TPP) cation, which is known to enter the mitochondrial matrix. ${ }^{2}$ Dhar had previously adapted the PGLA-PEG nanoparticle system to target prostate cancer cells. ${ }^{3}$

The Georgia team blended the resulting PGLA-PEG-TPP copolymer with a nontargeting PGLA-PEG copolymer to create the nanoparticles in a single-step process. This approach helped reduce the likelihood of unwanted variation in nanoparticle properties that normally occurs with an increase in the number of processing steps.

Finally, the researchers generated a series of nanoparticles with different sizes and surface charges by varying the relative amounts of targeting and nontargeting copolymers.

In a human cervical cancer cell line, nanoparticles containing the targeting copolymer showed greater uptake in mitochondria than nanoparticles that lacked the targeting copolymer. Nanoparticles 80-100 $\mathrm{nm}$ in diameter showed maximum uptake by mitochondria.

The researchers also showed that nanoparticles with positively charged surfaces had higher cellular and mitochondrial uptake as the surface charge increased, with maximum cellular uptake at $22 \mathrm{mV}$ and maximum mitochondrial uptake at $34 \mathrm{mV}$. and are produced using a single-step processing method," said Omid Farokhzad, associate professor at Harvard Medical School, founder of Blend Therapeutics Inc. and cofounder of Bind Biosciences Inc. and Selecta Biosciences Inc.

Bind's BIND-014, a polymeric nanoparticle that targets prostatespecific membrane antigen (PSMA; FOLH1; GCPII) and contains docetaxel, is in Phase I testing to treat advanced or metastatic solid tumors.

Dhar and Marrache next plan to use their nanoparticle system in vivo. "We will first test curcumin, lonidamine and 2,4-DNP in animal models," Dhar told SciBX. "If our results look promising, we will expand to other mitochondrial-acting therapeutics."

\section{Getting into the mitochondria}

Although the data in the PNAS paper show a marked improvement in mitochondrial targeting with the nanoparticles, actual selectivity and the mechanism underlying this effect remain to be determined.

"These nanoparticles, as creative as they are, have the main limitation that they will enter into all cells that they come into contact with," noted Erkki Ruoslahti, professor at the Sanford-Burnham Medical Research Institute and at the Center for Nanomedicine of the University of California, Santa Barbara. "After a systemic injection, the uptake 
would be mostly limited to the endothelial cells in blood vessels and the reticuloendothelial system. Getting the nanoparticles into extravascular tissue, where the target cells reside, will require additional solutions."

Ruoslahti said his lab has been working on nanoparticles that selectively target the mitochondria of tumor endothelial cells and tumor cells by incorporating a tumor-homing peptide. Compared with his approach, he said "Dhar's nanoparticles are more broadly applicable, which is both an advantage and a disadvantage."

Other researchers still had questions about the actual mechanism by which the nanoparticles interacted with mitochondria.

The researchers did not conclusively show "whether the nanoparticle passes across mitochondrial membranes," said Yuma Yamada, assistant professor of pharmaceutical sciences at Hokkaido University. "The nanoparticle most likely binds to the mitochondria selectively and releases the cargo at the mitochondrial site, with the compounds entering the mitochondrial compartment."

Mike Murphy, group leader of the Mitochondrial Biology Unit at the Medical Research Council, agreed. "The work has some very nice aspects, especially enhanced endosomal escape, but the researchers have not demonstrated actual targeting into mitochondria. Their characterization methods, such as confocal microscopy and cadmium assays, are inadequate to show this. They are probably seeing adsorption to the mitochondrial surface and not penetration to the mitochondrial matrix."

He added that Dhar's group would "need to show with isolated mitochondria that they are getting uptake into the matrix and then extend this to cell culture to show the same. There are well-established techniques to do this."

"We believe the particles are entering the mitochondria through the outer membrane and that the particles are located inside the mitochondrial matrix because the matrix-targeting particles are found in the mitochondrial fractions of cell culture-based assays," said Dhar. "Detailed in vitro studies are ongoing in the lab to study the nanoparticles' entry process and exact location in the mitochondria."

Alternatively, if the nanoparticles do not enter the matrix, Murphy said the system could potentially be "very useful because their biodegradable nanoparticles adsorb to the mitochondria, where they slowly break down to release the compounds."

In fact, according to Murphy, the nanoparticles described by Dhar could be used to deliver proteins or peptides near the mitochondria and thus "provide a breakthrough for slow delivery inside cells in general."

The University of Georgia has filed for a patent covering the work, and the IP is available for licensing.

Baas, T. SciBX 5(38); doi:10.1038/scibx.2012.998

Published online Sept. 27, 2012

\section{REFERENCES}

1. Marrache, S. \& Dhar, S. Proc. Natl. Acad. Sci. USA; published online Sept. 18, 2012; doi:10.1073/pnas.1210096109

Contact: Shanta Dhar, The University of Georgia, Athens, Ga. e-mail: shanta@uga.edu

2. Smith, R.A.J. et al. Proc. Natl. Acad. Sci. USA 100, 5407-5412 (2003)

3. Kolishetti, N. et al. Proc. Natl. Acad. Sci. USA 107, 17939-17944 (2010)

COMPANIES AND INSTITUTIONS MENTIONED

Bind Biosciences Inc., Cambridge, Mass.

Blend Therapeutics Inc., Watertown, Mass.

Harvard Medical School, Boston, Mass.

Hokkaido University, Sapporo, Japan

Medical Research Council, Cambridge, U.K.

Sanford-Burnham Medical Research Institute, La Jolla, Calif.

Selecta Biosciences Inc., Watertown, Mass.

Stanford University, Stanford, Calif.

The University of Georgia, Athens, Ga.

University of California, Santa Barbara, Calif. 